\title{
SQUID-Detected Magnetic Resonance Imaging in Microtesla Fields
}

\author{
Michael Mößle, Michael Hatridge and John Clarke \\ Department of Physics, University of California, Berkeley CA 94720-7300 \\ and
}

Materials Sciences Division, Lawrence Berkeley National Laboratory, Berkeley CA 94720

Running title: $\quad$ SQUID-detected MRI

Corresponding author: John Clarke

Physics Department

University of California/Berkeley

Berkeley, CA 94720-7300

Phone: (510) 642-3069 Fax: (510) 642-1304

Email: jclarke@berkeley.edu 


\section{INTRODUCTION}

Magnetic resonance imaging (MRI) has developed into a powerful clinical tool for imaging the human body (1). This technique is based on nuclear magnetic resonance (NMR) of protons (2, 3) in a static magnetic field $B_{0}$. An applied radiofrequency pulse causes the protons to precess about $B_{0}$ at their Larmor frequency $v_{0}=(\gamma / 2 \pi) B_{0}$, where $\gamma$ is the gyromagnetic ratio; $\gamma / 2 \pi=$ 42.58 MHz/tesla. The precessing protons generate an oscillating magnetic field and hence a voltage in a nearby coil that is amplified and recorded. The application of three-dimensional magnetic field gradients specifies a unique magnetic field and thus an NMR frequency in each voxel of the subject, so that with appropriate encoding of the signals one can acquire a complete image (4). Most clinical MRI systems involve magnetic fields generated by superconducting magnets, and the current trend is to higher magnetic fields than the widely used 1.5-T systems (5). Nonetheless, there is ongoing interest in the development of less expensive imagers operating at lower fields. Commercially available 0.2 -T systems based on permanent magnets offer both lower cost and a more open access than their higher-field counterparts, at the expense of signal-to-noise-ratio (SNR) and spatial resolution. At the still lower field of $0.03 \mathrm{mT}$ maintained by a conventional, room-temperature solenoid, Connolly and co-workers $(6,7)$ obtain good spatial resolution and signal-to-noise ratio (SNR) by prepolarizing the protons in a field $B_{p}$ of $0.3 \mathrm{~T}$. Prepolarization (8) enhances the magnetic moment of an ensemble of protons over that produced by the lower precession field; after the polarizing field is removed, the higher magnetic moment produces a correspondingly larger signal during its precession in $B_{0}$. Using the same method, Stepisnik et al. (9) obtained MR images in the Earth's magnetic field $(\sim 50 \mu \mathrm{T})$. Alternatively, one can enhance the signal amplitude in MRI using laser polarized noble gases such as ${ }^{3} \mathrm{He}$ or ${ }^{129} \mathrm{Xe}(10-12)$. 
Hyperpolarized gases were used successfully to image the human lung in fields on the order of several mT (13-15).

To overcome the sensitivity loss of Faraday detection at low frequencies, ultrasensitive magnetometers based on the Superconducting QUantum Interference Device (SQUID) (16) are used to detect NMR and MRI signals (17-24). Recently, SQUID-based MRI systems capable of acquiring in vivo images have appeared. For example, in the 10-mT system of Seton et al. (18) signals are coupled to a SQUID via a superconducting tuned circuit, while Clarke and coworkers $(22,25,26)$ developed a system at $132 \mu \mathrm{T}$ with an untuned input circuit coupled to a SQUID. In a quite different approach, atomic magnetometers have been used recently to detect the magnetization (27) and NMR signal (28) of hyperpolarized gases. This technique could potentially be used for low-field MRI in the future.

The goal of this review is to summarize the current state-of-the-art of MRI in microtesla fields detected with SQUIDs. The principles of SQUIDs and NMR are briefly reviewed. We show that very narrow NMR linewidths can be achieved in low magnetic fields that are quite inhomogeneous, with illustrative examples from spectroscopy. After describing our ultralow-field MRI system, we present a variety of images. We demonstrate that in microtesla fields the longitudinal relaxation $T_{1}$ is much more material dependent than is the case in high fields; this results in a substantial improvement in " $T_{1}$-weighted contrast imaging." After outlining the first attempts to combine microtesla NMR with magnetoencephalography (MEG) (29), we conclude with a discussion of future directions. 


\section{BASIC PRINCIPLES}

\section{The Superconducting QUantum Interference Device}

In the phenomenon of superconductivity (30), pairs of electrons - known as "Cooper pairs"carry a supercurrent without dissipation. The de SQUID (16) combines two phenomena of the superconducting state: Josephson tunneling and flux quantization (30). In a Josephson junction, Cooper pairs of electrons tunnel coherently through a thin barrier, typically a metal oxide. The junction remains in the zero voltage state for applied currents up to a critical value, above which a voltage is produced. Flux quantization refers to the fact that the magnetic flux embraced by a closed superconducting loop is quantized in units of the flux quantum $\Phi_{0}=h / 2 \mathrm{e}$ $\approx 2.07 \times 10^{-15} \mathrm{Tm}^{2} ; \mathrm{h}$ is Planck's constant and $\mathrm{e}$ is the electron charge. The SQUID consists of two Josephson junctions connected in parallel on a superconducting loop [Figure 1(a)]. When the magnetic flux $\Phi$ threading the loop is steadily changed, the current-voltage $(I-V)$ characteristic oscillates back and forth between the two values shown in Figure 1(b) with a period $\Phi_{0}$. In the presence of a constant bias current, the voltage across the SQUID oscillates as a function of $\Phi$, as shown in Figure 1(c). The SQUID is usually operated at a flux where a small change in magnetic flux $\delta \Phi$ produces the maximum change in voltage $\delta V$. The voltage $\delta V$ is amplified by conventional semiconductor electronics; in essence, the SQUID is a flux-tovoltage transducer. Design rules for optimizing the performance of the SQUID are well established (31).

SQUIDs are fabricated from thin films using the deposition and patterning techniques of the semiconductor industry. An example is shown in Figure 2(a); typically 200-400 such devices are made on a 4-inch silicon wafer. The SQUID is generally operated immersed in liquid helium at $4.2 \mathrm{~K}$. In practice, the SQUID is operated in a flux-locked loop (16) in which 
the voltage $\delta V$ is amplified and the resultant signal is coupled via a resistance into a coil inductively coupled to the SQUID. This negative feedback maintains the flux in the SQUID at a constant value, and results in an output voltage from the electronics that scales linearly with the input flux signal. Typical devices have a magnetic flux noise of about $1 \mu \Phi_{0} \mathrm{~Hz}^{-1 / 2}$. In many applications the sensitivity to magnetic field is enhanced by a superconducting flux transformer [Figure 2(b)], which consists of a loop of superconducting wire connected to a multiturn coil deposited on (but insulated from) the SQUID [Figure 2(a)]. When a magnetic field is applied to the pickup loop, flux quantization causes a supercurrent to flow around the closed superconducting circuit, coupling flux into the SQUID. An optimized flux transformer can yield a magnetic field noise of $1 \mathrm{fT} \mathrm{Hz}^{-1 / 2}\left(10^{-15} \mathrm{~T} \mathrm{~Hz}^{-1 / 2}\right)$. Figure 3 depicts the scale of magnetic fields involved in SQUID magnetometers and MRI. We emphasize that the responses of the SQUID and flux transformer are independent of the signal frequency.

\section{Nuclear Magnetic Resonance of Protons}

In the presence of a magnetic field $B_{0}$, a spin $1 / 2$ particle - for example a proton - aligns itself parallel or antiparallel to the field with corresponding energies $-\gamma \hbar B_{0} / 2$ and $+\gamma \hbar B_{0} / 2$, respectively. The angular frequency corresponding to transitions between the two levels is the Larmor frequency $\omega_{0}=\gamma B_{0}$. For an ensemble of $\mathrm{N}$ noninteracting protons in thermal equilibrium in a field $B_{0}$ at a temperature $T$, the magnetic moment is $M_{0}=N \mu_{p}^{2} B_{0} / \mathrm{k}_{\mathrm{B}} T$ in the relevant limit $\mu_{p} B_{0}<<\mathrm{k}_{\mathrm{B}} T ; \mu_{p}$ is the magnetic moment of the proton and $\mathrm{k}_{\mathrm{B}}$ is Boltzmann's constant. The fractional magnetization is generally very small: at $300 \mathrm{~K}$ and $1 \mathrm{~T}, M_{0} / N \mu_{p} \approx 3.4$ $\mathrm{x} 10^{-6}$. 
Virtually all NMR experiments involve the application of pulsed magnetic fields. In the presence of a static magnetic field in the z-direction, one applies a " $\pi / 2$ pulse" of oscillating magnetic field $B_{1} \cos \omega_{0} t$ in the y-direction with the appropriate amplitude and duration to tip $M_{0}$ into the x-y plane. Subsequently, $M_{0}$ precesses about the z-axis at frequency $\omega_{0} / 2 \pi$. During the precession, $M_{0}$ is subject to two relaxation processes. The first is relaxation of its direction towards the z-axis, along which it will ultimately regain equilibrium, in the longitudinal or spin-lattice relaxation time $T_{1}$. The second process is the dephasing of each proton spin by the fluctuating local field produced at its site by neighboring spins in the transverse or spin-spin relaxation time $T_{2}$. In tissue, $T_{1}$ and $T_{2}$ range from 10's of milliseconds to about $1 \mathrm{sec}$. The relaxation time $T_{2}$ determines the observed NMR linewidth $\Delta v=1 / \pi T_{2}$ ("homogeneous broadening"). An inhomogeneous precession field, however, can substantially broaden the linewidth since protons in different parts of the sample are exposed to different magnetic fields. This inhomogeneous broadening, described by the inhomogenous lifetime $T_{2}{ }^{\prime}$, reduces the effective transverse relaxation time to $T_{2}{ }^{*}=\left(1 / T_{2}+1 / T_{2}{ }^{\prime}\right)^{-1}$.

Most NMR spectra are obtained using Hahn's spin echo technique (32). At time $\tau$ after the $\pi / 2$ pulse, a $\pi$ pulse is applied along the $y$-axis, causing $M_{0}$ to flip through $180^{\circ}$. In their subsequent precession, the spins refocus to produce an "echo" at a time $2 \tau$ after the $\pi / 2$ pulse. Only inhomogeneous broadening is reduced by the spin echo; homogeneous broadening, which is due to randomly fluctuating spins, is unaffected.

\section{Why Use a SQUID?}

In conventional NMR and MRI, the precessing magnetic moment induces a voltage in a nearby coil with a magnitude that, by Faraday's law, scales as $\omega_{0} M_{0}$; since $M_{0}$ also scales as $\omega_{0}$, the 
voltage scales as $\omega_{0}^{2}$ or $B_{0}{ }^{2}$. This scaling is a major incentive to increase the static field. By the same token, as one reduces the field, the amplitude of the voltage falls off quadratically with $B_{0}$, making very low field Faraday-detected NMR or MRI unattractive. There are two factors, however, that mitigate this loss of signal at low fields. First, SQUIDs respond to the field itself rather than the rate of change of field; consequently, the output voltage scales as $M_{0}$ or $\omega_{0}$, rather than $\omega_{0}^{2}$ as for Faraday detection. Second, if one prepolarizes the sample in a field $B_{p}$ greater than $B_{0}(8)$, the magnetic moment so induced is independent of and greater than its value in $B_{0}$. As a result, one is free to choose $B_{0}-$ subject to other constraints - without sacrificing signal amplitude. The combination of SQUID-detection and prepolarization enables microtesla NMR and MRI.

An additional benefit is immediately apparent. The inhomogeneous linewidth $\Delta v^{\prime}$ is given by $(\gamma / 2 \pi) \Delta B_{0}=(\gamma / 2 \pi)\left(\Delta B_{0} / B_{0}\right) B_{0}$, where $\Delta B_{0}$ is the net change in $B_{0}$ over the sample. Consequently, for the given fractional inhomogeneity $\Delta B_{0} / B_{0}$ of a particular set of coils, the linewidth scales as $B_{0}$. As an example, to achieve a linewidth of $1 \mathrm{~Hz}$ in a field of $20 \mathrm{~T}$ (proton Larmor frequency $860 \mathrm{MHz}$ ), one requires a magnetic field homogeneity of about 1 part in $10^{9}$, whereas to achieve the same linewidth in the Earth's field, about $50 \mu \mathrm{T}$ (proton Larmor frequency about $2 \mathrm{kHz}$ ), one requires a homogeneity of only 1 part in 2000 . Thus, lowering the magnetic field leads to much less stringent requirements on the magnetic field homogeneity. By the same token, the effect of inhomogeneities introduced into the static magnetic field by variations in the magnetic susceptibility of the sample also scale as $B_{0}$, and are likely to be of no consequence at microtesla fields. 


\section{MICROTESLA NUCLEAR MAGNETIC RESONANCE}

In this section, we demonstrate the narrowing of NMR linewidths as the magnetic field produced by a given set of coils is reduced. Experiments (21) were carried out on mineral oil maintained near room temperature in a double-walled glass tube immersed in liquid helium. A relatively inhomogeneous precession field $B_{0}$ was supplied by a small Helmholtz pair, and an orthogonal polarizing field $B_{p}$ was pulsed on and off. The signal produced by the precessing protons in the mineral oil in a direction perpendicular to $B_{0}$ was detected by a flux transformer coupled to a SQUID.

Figures 4(a) and (b) summarize the central result. Figure 4(a) shows the NMR spectrum of mineral oil acquired in a field of $1.8 \mathrm{mT}$ using a Hahn spin echo sequence. The proton peak at about $77 \mathrm{kHz}$ has a linewidth of about $1 \mathrm{kHz}$, indicating a magnetic field inhomogeneity $\Delta B_{0} / B_{0}$ of about $1 \%$. In Figure $4(\mathrm{~b})$, on the other hand, the static field $B_{0}$ was reduced by three orders of magnitude to $1.8 \mu \mathrm{T}$, and the protons were prepolarized in a magnetic field of $1.8 \mathrm{mT}$. The polarizing field was removed before the data were acquired. We immediately observe the two consequence of reducing $B_{0}$. First, the NMR frequency has been reduced by three orders of magnitude, to $77 \mathrm{~Hz}$. Second, the linewidth has been reduced, also by three orders of magnitude, to about $1 \mathrm{~Hz}$, verifying its scaling with $B_{0}$. Furthermore, while the spectrum in Figure 4(a) required 10,000 averages that in Figure 4(b) required only 100 averages and has a much higher SNR. Because the value of $B_{p}$ in (b) was identical to $B_{0}$ in (a), the energy under the two peaks is the same and the reduction of the linewidth in (b) results in an enhancement in the peak height by the same factor.

The enhanced spectral resolution achieved in microtesla fields was exploited to detect scalar coupling (“J-coupling”) in heteronuclear spin systems. $(21,33)$. Figure 5(a) shows the 
spectrum of neat trimethyl phosphate in a field of $4.8 \mu \mathrm{T}$. The doublet is formed by the interaction of the nine equivalent protons with the single phosphorus ${ }^{31} \mathrm{P}$ nucleus. The splitting of $10.4 \pm 0.6 \mathrm{~Hz}$ arises from the tiny difference in energy depending on whether the proton spins are parallel or antiparallel to the phosphorus spin. The linewidth was limited by the inhomogeneity in the residual magnetic field in the apparatus in which the data were acquired. A further reduction in linewidth was achieved $(\mathrm{P})(24,34)$ in the magnetically shielded room at the Physikalisch Technische Bundesanstalt (PTB) in Berlin (35) by subtracting the output of two SQUID magnetometers electrically to form a first-derivative gradiometer; the very low $(<10 \mathrm{nT})$ residual field enabled Burghoff et al. (24) to achieve a linewidth of $0.17 \mathrm{~Hz}$ in distilled water in a field of $450 \mathrm{nT}$ corresponding to a Larmor frequency of about $19 \mathrm{~Hz}$. Subsequently, using the same system Bernarding et al. (34) obtained the J-coupling spectrum of 2, 2, 2-trifluoroethanol shown in Figure 5(b). The spectra have been fitted with a $T_{2}$ of 1 sec, corresponding to a linewidth of $0.32 \mathrm{~Hz}$.

These very narrow linewidths achieved in microtesla magnetic fields that are grossly inhomogeneous by the standards of high field NMR and MRI show that simple and inexpensive magnets are entirely adequate for microtesla spectroscopy and imaging. High spectral resolution NMR leads immediately to high spatial resolution $\Delta z$ in MRI, since $\Delta z=$ $2 \pi \Delta v / \gamma G_{z}$ where $G_{z} \equiv \partial B_{z} / \partial z$ is an applied gradient The determination of J-coupling spectra by direct detection of the NMR spectrum could be used as a "bond detector," for example, to monitor chemical reactions or to identify specific chemical compounds in blood samples. 


\section{MAGNETIC RESONANCE IMAGING IN MICROTESLA MAGNETIC FIELDS Experimental Configuration and Techniques}

The essential requirements of conventional MRI are a magnet to produce a precession field $B_{0}$, three sets of gradient coils, a transmitter coil to supply the necessary pulse sequences and a receiver. Microtesla MRI involves the same set of coils, albeit with greatly reduced requirements on the static field and gradient coils and a low-frequency receiver; in addition, a polarizing field coil and coils to cancel the Earth's magnetic field are required. As an example, Figure 6 shows the arrangement of coils in the Berkeley microtesla MRI system. The coils are wound on wooden forms assembled on a wooden frame. Two pairs of square coils $1.8 \mathrm{~m}$ on a side cancel the $x$ - and $y$-components of the Earth's magnetic field. A 1.37-m-diameter Helmholtz pair, augmented by a pair of concentric $0.36-\mathrm{m}$-diameter coils in the same planes to increase the field homogeneity, supplements the Earth's field in the $z$-direction to provide the precession field $B_{0}$. The NMR frequency is usually $5.6 \mathrm{kHz}$, corresponding to $B_{0}=131.5 \mu \mathrm{T}$. This frequency lies between two harmonics of $60 \mathrm{~Hz}$ and is in a range where the background noise in our laboratory is low, typically $1 \mathrm{pT} \mathrm{Hz}^{-1 / 2}$. A Maxwell pair of coils, $1.2 \mathrm{~m}$ in diameter, provides the diagonal field gradient $\partial B_{z} / \partial z$, and two sets of biplanar coils $(0.9 \times 1.28 \mathrm{~m}$ and $1.45 \times 1.02 \mathrm{~m})$ provide the off-diagonal gradients $\partial B_{z} / \partial x$ and $\partial B_{z} / \partial y$. Typical field gradients range from 100 to $400 \mu \mathrm{T} \mathrm{m}^{-1}$. The excitation coil consists of a $0.155-\mathrm{m}$-diameter Helmholtz pair that produces field pulses along the y-direction. The polarizing coil produces pulsed fields of up to150 mT in the $x$-direction. The polarizing coil involves a substantial mass of copper wire; if this wire were solid, Nyquist noise currents flowing across the wire would induce significant levels of magnetic field noise at the nearby detector. Fortunately, these fields can be reduced to a negligible level by using multistranded wire - we use 30-strand, 28 gauge Litz 
wire - since the noise amplitude scales as the square of the wire diameter (36). The polarizing coil currently installed in our system consists of 700 turns of Litz wire and has an inner diameter of $32 \mathrm{~mm}$, an outer diameter of $230 \mathrm{~mm}$ and a height of $50 \mathrm{~mm}$. To increase the number of coil pulses this coil is precooled with liquid nitrogen. Samples are placed on top of the polarizing coil, below the detector.

The NMR signals from the sample are detected by a flux transformer, configured as a second-derivative axial gradiometer and coupled to a dc SQUID as shown schematically in Figure 7. Ideally, the gradiometer rejects ambient fluctuations in uniform magnetic fields and its first derivatives, and responds only to the diagonal gradient $\partial^{2} B_{z} / \partial z^{2}$. Because the field and its first and second derivatives fall off with distance $r$ from a magnetic dipole as $1 / r^{3}, 1 / r^{4}$ and $1 / r^{5}$, respectively, the use of a second-derivative gradiometer greatly reduces the magnitude of magnetic noise from distant sources. As fabricated, the gradiometer attenuates uniform magnetic field fluctuations by a factor of about 1,000 . The sample to be imaged is placed close to the lowest loop of the gradiometer, which thus detects the magnetic field from the sample, rather than the second-derivative gradient. The magnetic field noise of the detector referred to the lower loop is $0.8 \mathrm{fT} \mathrm{Hz}^{-1 / 2}$. An important feature is the array of 20 Josephson junctions in series with the gradiometer coils. The magnetic fields applied with the various coils (especially the polarizing coil) would otherwise induce enormous currents (up to $1 \mathrm{~A}$ ) in the gradiometer. The Josephson junctions switch to the normal state, however, when the induced current exceeds their critical current, typically $10 \mu \mathrm{A}$, thus preventing any further build-up of current. Once the magnetic field becomes stable, the junctions revert to their superconducting state. The SQUID and the array are contained in a superconducting niobium shield to protect them from external magnetic noise and the switched fields of the MRI procedure. 
The flux transformer and SQUID are immersed in liquid helium contained in a custommade fiberglass dewar based on a design by Seton et al. (37). In essence, the dewar consists of an inner vessel, containing the helium, surrounded by an outer vessel with a vacuum space between them. To prevent the blackbody radiation from the outer vessel imposing an unacceptable heat load on the helium, it is conventional to fill the vacuum space with many layers of aluminized Mylar that reflect the radiation. The sensitivity of the detector is sufficiently high, however, that magnetic field noise originating in Nyquist noise currents in the thin aluminum layers would seriously degrade its performance. Seton and co-workers overcame this problem by replacing the aluminized Mylar with aluminized polyester fabric. The weave of the fabric breaks up continuous conducting paths, thus preventing the flow of noise currents over large areas and reducing the magnetic field noise by as much as two orders of magnitude compared with a continuous aluminum film with the same thickness.

The entire system is enclosed in a cube, $2.4 \mathrm{~m}$ on a side, constructed of 6-mm-thick aluminum sheet. Eddy currents in the sheet reduce external electromagnetic fields to 1/e of their surface value in a skin depth $\delta=\left(\pi \mu_{0} \mu \sigma f\right)^{-1 / 2} ; \mu_{0}$ is the permeability of the vacuum, and $\mu$ and $\sigma$ are the relative permeability and electrical conductivity of the material. For aluminum at $5.6 \mathrm{kHz}, \delta \approx 1.5 \mathrm{~mm}$, so that the shield attenuates external noise by a factor of about $\mathrm{e}^{4} \approx 55$. The combination of the aluminum shield and the second-derivative gradiometer attenuates ambient magnetic field fluctuations by a factor of about $5 \times 10^{4}$, so that ambient noise does not affect the measurements.

A typical pulse sequence for three-dimensional (3-D) imaging is shown in Figure 8. The static field $B_{0}$ is applied continuously. First, the polarizing field $B_{p}$ is turned on along the $x$-axis for a time typically on the order of $T_{1}$, and removed in $15 \mathrm{~ms}$. A $\pi / 2$ pulse of $5.6 \mathrm{kHz}$ 
magnetic field is applied along the $y$-direction, and is followed by a $\pi$ pulse a time $\tau$ later. Shortly after the $\pi / 2$ pulse, three gradient pulses are applied. The gradient $G_{x} \equiv \partial G_{z} / \partial x$ is applied during the whole measurement and encodes voxels in frequency space as

$$
\omega(x)=\gamma\left(B_{0}+x G_{x}\right) .
$$

The gradients $G_{y} \equiv \partial B_{z} / \partial y$ and $G_{z} \equiv \partial B_{z} / \partial z$, on the other hand, are applied only between the $\pi / 2$ and $\pi$ pulses and provide phase encoding according to

$$
\Delta \phi(z)=\gamma z G_{y} \tau, \quad \Delta \phi(y)=\gamma y G_{z} \tau .
$$

The last line in Figure 6 shows the free induction decay induced by the $\pi / 2$ pulse and the echo resulting from the subsequent $\pi$ pulse. The spatial information is frequency- and phaseencoded in the echoes, which are digitized and stored for subsequent processing. Twodimensional images are acquired by omitting one of the phase encoding sequences.

\section{Images}

We present a series of 2-D and 3-D images to illustrate the capabilities of the 132- $\mu \mathrm{T}$ imager. Figure 9 shows a 2-D image of a phantom consisting of a plastic multiwell plate with $0.85 \mathrm{~mm}$ walls immersed in $10 \mathrm{~mm}$ of water in a plastic tray. The image was acquired at $132 \mu \mathrm{T}$ (proton Larmor frequency $5.6 \mathrm{kHz}$ ) using a polarizing field of $85 \mathrm{mT}$. The frequency encoding gradient was $240 \mu \mathrm{T} \mathrm{m}^{-1}$ and there were 128 phase encoding steps with a maximum gradient

of $140 \mu \mathrm{T} \mathrm{m}^{-1}$. The 2-D resolution is $0.7 \mathrm{~mm} \times 0.7 \mathrm{~mm}$ and the total imaging time was $\sim 5 \mathrm{~min}$. To illustrate 3-D imaging, Figure 9 shows 6 MRI slices of an intact red pepper. In contrast to the multi-slice echo-planar imaging technique of high-field MRI (4), because of the time required to polarize the spins it is more efficient to acquire the entire 3-D image and to slice the image subsequently. The image involved a frequency encoding gradient of $150 \mu \mathrm{T} / \mathrm{m}, 52$ 
phase encoding steps in the plane of the slices and 7 phase encoding steps perpendicular to the slices. The polarizing field was $60 \mathrm{mT}$ and the precession field was $132 \mu \mathrm{T}$. The resolution of the image is $1.9 \mathrm{~mm} \times 1.6 \mathrm{~mm}$.

Figure 10 shows four MR slices of a forearm, each 20-mm-thick. This in vivo image was acquired at $5.6 \mathrm{kHz}$ with gradients of $150 \mu \mathrm{T} / \mathrm{m}$ and an average polarizing field of $40 \mathrm{mT}$. The in-plane resolution is $2 \mathrm{~mm} \times 2 \mathrm{~mm}$ and the SNR is 10 .

The images presented in Figures 9 and 10 illustrate the current state-of-the-art of microtesla MRI. In the images of the phantom, a 2-D resolution of about $1 \mathrm{~mm} \times 1 \mathrm{~mm}$ is relatively straightforward. The spatial resolution of the arm, about $2 \mathrm{~mm} \times 2 \mathrm{~mm}$, is lower largely because the configuration of our current polarizing coil results in a rapid fall-off in the polarizing field across the arm; this problem could be ameliorated by replacing the single-sided coil with a Helmholtz pair. In all cases, by the standards of high-field MRI, the imaging process is relatively slow, with acquisition times ranging from $1 \mathrm{~min}$ for the phantom to $5 \mathrm{~min}$ for the three slices of the forearm. These times are dictated primarily by the time required to polarize the protons for a given number of phase encoding steps.

\section{Imaging in the Presence of Metals}

In high-field MRI, images acquired in the presence of a piece of metal, for example, an orthopedic screw or a biopsy needle, are often severely distorted (38-40). The distortion has two possible origins. The first is the susceptibility difference between the metal and the surrounding tissue which causes a local magnetic field inhomogeneity proportional to $B_{0}$, and is therefore negligible at microtesla fields. In turn, this inhomogeneity both shortens $T_{2}{ }^{*}$ and produces image distortion along the frequency encoding direction. The second mechanism is 
the interaction of the metal object with the excitation pulses, which induce eddy currents in its skin depth. These eddy currents can affect the amplitude and phase of the pulse near the metal, giving rise to distortion. Since the skin depth $\delta$ scales as $f_{0}^{-1 / 2}$, this problem is substantially reduced at low frequencies.

To illustrate these issues, in Figure 11 we show images of a $1.5 \mathrm{~mm}$ x $10 \mathrm{~mm} \times 15 \mathrm{~mm}$ titanium bar placed in one of a series of grooves cut in a plastic block to form a grid; the grid is immersed in water (41). Figure 11(a) shows a photograph of the phantom used at $7 \mathrm{~T}$, and Figures 11(b) and (c) show the resulting images acquired using a gradient of $40 \mathrm{mT} \mathrm{m}^{-1}$ with the bar oriented perpendicular and parallel to the frequency-encoding direction. In both cases, the images are severely distorted. In contrast, Figure 11(e) is the image of the phantom shown in Figure 11(d) acquired at $2.8 \mathrm{kHz}$ with a gradient of $110 \mu \mathrm{T} \mathrm{m}^{-1}$. There is no evident distortion. These results suggest that microtesla MRI could be used to obtain undistorted images in the presence of metallic implants or biopsy needles.

In related experiments at $2.8 \mathrm{kHz}$, images were obtained of a pepper enclosed in an aluminum can with a wall thickness of $200 \mu \mathrm{m}$ (41). Because the wall thickness was much less than the $1.5-\mathrm{mm}$ skin depth, the quality of the image was unaffected by the presence of the can. In contrast, attempts to obtain images at $179 \mathrm{MHz}$ failed because the 6- $\mu \mathrm{m}$ skin depth screened out the radiofrequency pulses and the signal.

\section{RELAXATION-TIME-WEIGHTED CONTRAST IMAGES}

$T_{1}$-weighted contrast imaging is widely used in high-field MRI to obtain image contrast between tissues with different longitudinal relaxation times $T_{1}(4)$. In practice, however, not all tissue types show $T_{1}$-contrast in high fields; for example, breast tumors are not readily 
distinguished from healthy breast tissue (42). This difficulty can be overcome by injecting a contrast agent - typically a gadolinium salt - into the bloodstream which tends to flow preferentially to the tumor $(43,44)$. The contrast agent reduces $T_{1}$, resulting in clear images of the tumor. For most tissues $T_{1}$ depends strongly on the magnetic field strength, and the $T_{1}$ contrast between different tissue types can be enhanced at low magnetic fields. Prepolarized SQUID-detected MRI enables the magnetization to evolve at very low fields, and could therefore be used for $T_{1}$-contrast imaging without the need for a contrast agent. To elucidate the issues involved, we briefly discuss the underlying mechanisms that determine the value of $T_{1}$.

The magnitude of $T_{1}$ is determined by the spectral density of the local magnetic field fluctuations at the NMR frequency (2). Random fluctuations caused by molecular motion and chemical exchange occurring at the correlation time $\tau_{c}$ have strong spectral components up to a frequency $f_{c}=1 / 2 \pi \tau_{c}$. Although in pure water $\tau_{c} \sim 1 \mathrm{ps}$ for protons, in tissue and macromolecular solutions $\tau_{c}$ is very much longer, $1 \mu \mathrm{s}$ to $1 \mathrm{~ms}$. Consequently, at frequencies below $1 / \tau_{c}$ the value of $T_{1}$ is sensitive to the molecular environment - and hence to the type of tissue - while at frequencies above $1 / \tau_{c}$ the values of $T_{1}$ in different tissues tend to converge because the proton spins decouple from the relaxation mechanisms that are specific to the environment.

To investigate the $T_{1}$ relaxation over a large range of magnetic fields we used dilute solutions of agarose gel as a model system. In this case one can express the overall proton relaxation rate as the sum of the two terms, one for free water and other for water molecules bound to the gel (45), as

$$
1 / T_{1}=(1-b) / T_{1 f}+b / T_{1 b} .
$$


Here, $b$ is the fraction of bound water (typically $<1 \%$ ) and $T_{1 f}$ and $T_{1 b}$ are the relaxation times of free and bound water molecules. The relaxation rate $1 / T_{1 f}$ is independent of frequency up to very high frequencies, but $1 / T_{1 b}$ increases sharply at frequencies below $1 / \tau_{c b} ; \tau_{c b}$ is the correlation time for bound water molecules. Consequently, the bound fraction of the water dominates the observed relaxation rate $1 / T_{1}$ at frequencies below $1 / \tau_{c b}$. Thus, one expects that low-frequency $T_{1}$-contrast should be substantially enhanced compared with high-field MRI.

This behavior was explored using the MRI system described above (46). The ability to cycle the magnetic field over a wide range of values made it straightforward to measure $T_{1}$ over the range $1 \mu \mathrm{T}$ to $300 \mathrm{mT}$. The pulse sequence is shown in Figure 12(a). The samples are first polarized in a magnetic field $B_{p 1}$ of $300 \mathrm{mT}$. To measure $T_{1}$ in fields greater than $132 \mu \mathrm{T}$, the polarizing field is reduced adiabatically to $B_{p 2}$ thus producing an intermediate field $\boldsymbol{B}_{\text {int }}=$ $\boldsymbol{B}_{p 2}+\boldsymbol{B}_{0}$ in which the spins relax for time $t_{i n t}$. Subsequently, $B_{p 2}$ is switched off adiabatically, causing the spins to align along $B_{0}$ with a magnetization

$$
M_{1}=M_{\text {int }}+\left(M_{p 1}-M_{\text {int }}\right) \exp \left(-t_{\text {int }} / T_{1 \text { int }}\right) .
$$

Here, $M_{\text {int }}$ and $M_{p 1}$ are the magnetic moments in $B_{\text {int }}$ and $B_{p 1}$, respectively, and $T_{1 \text { int }}$ is the longitudinal relaxation time in $B_{\text {int }}$. The value of $M_{1}$, which determines the intensity of the image, is measured with a conventional spin-echo sequence. If $B_{\text {int }}=B_{0}$, the pulse sequence measures $T_{1}$ in the field $B_{0}$ during the time $t_{\text {int }}$. To measure $T_{1}$ in fields $B_{\text {int }}<B_{0}$, the value of $B_{0}$ is reduced for the time $t_{\text {int }}$ by applying an opposing field from the pair of Earth's field cancellation coils aligned along the $z$-axis. At a given intermediate field, $T_{1}$ is obtained by measuring the signal amplitude for increasing values of $t_{i n t}$. 
The resulting values of $1 / T_{1}$ versus magnetic field and Larmor frequency are plotted in Figure 12 (b) for $0.25 \%$ and $0.5 \%$ concentrations by weight of agarose gel in water. The solid curves are fits to the Cole-Cole expression (47)

$$
1 / T_{1}(f)=A \cdot \operatorname{Re}\left\{1 /\left[1+\left(\mathrm{i} f f f_{c}\right)^{\beta / 2}\right]\right\}+B,
$$

where $A, B, f_{\mathrm{c}}$ and $\beta$ are fitting parameters. The fits yield $\tau_{c b}$ of $3 \mu$ s and $2 \mu$ s for the $0.25 \%$ and $0.5 \%$ gels, respectively. At frequencies below $1 / \tau_{c b}$, the relaxation rates differ by a factor of about 2, while at higher frequencies the two curves converge to the rate for free water. These results dramatically illustrate the greatly enhanced $T_{1}$-contrast obtainable at low fields.

To demonstrate the impact of these results on an image, we prepared a sample consisting of 9 plastic drinking straws, each filled with water, arranged in a plastic tube containing $0.5 \%$ agarose gel [Figure 12(c)]. The inner diameters of the straws ranged from $1 \mathrm{~mm}$ to $6 \mathrm{~mm}$. To obtain images, the prepolarization and relaxation sequence of Figure 12(a) was followed by a 2-D imaging sequence. The lower left image shows an image taken with an intermediate magnetic field value of $100 \mathrm{mT}$, and shows virtually no contrast; the 6-mm water column is just discernible. At $132 \mu \mathrm{T}$, on the other hand, all the water columns are clearly visible. These two images dramatically demonstrate the benefit of $T_{1}$-contrast imaging at low magnetic fields.

High-field MRI often uses "inversion recovery" to highlight $T_{1}$-weighted contrast (4). A related technique, originally called "magnetization subtraction" (48), can be used to enhance the low-field contrast. Figure 13(a) shows the magnetization preparation for a method dubbed "field-cycling inversion recovery" (46). As before, the spins are first polarized in a strong field $B_{p 1}$. This field is removed adiabatically to allow the spins to align along $B_{0}$, in which they relax for a time $t_{\text {int }}$. For two spin populations with different values of $T_{1}$, the magnetic 
moments differ after this interval. Subsequently, a $\pi$ pulse inverts the populations and immediately afterwards a second polarization field $B_{p 2}$ is switched on adiabatically. The inverted populations recover towards thermal equilibrium in $B_{p 2}$; in so doing, they pass through zero magnetization at different times. Finally, the field is switched adiabatically to $B_{0}$ and the imaging sequence is implemented. The magnitude of the NMR signal depends critically on the time at which $B_{p 2}$ is removed and the imaging sequence is initiated. If the sequence begins when the magnetic moment of a given population is precisely zero, the resulting image will appear black. Thus, by choosing the onset of imaging appropriately, one can make one or other of the populations "vanish" in the image. Field-cycling inversion recovery is thus a powerful tool to enhance $T_{1}$-weighted contrast compared to standard inversion recovery sequences at high magnetic fields.

Figure 13(b) shows images of three glass tubes filled with tap water, $0.25 \%$ agarose gel and $0.5 \%$ agarose gel, respectively, obtained with the field-cycling inversion image sequence of Figure 13(a) followed by a 2D imaging sequence. Whereas all three samples have almost the same relaxation time, $1.8 \pm 0.1 \mathrm{sec}$ in $B_{p 1}=B_{p 2}=300 \mathrm{mT}$, at $132 \mu \mathrm{T}$ the values of $T_{1}$ are $1.6 \mathrm{sec}, 0.48 \mathrm{sec}$ and $0.21 \mathrm{sec}$ for water, $0.25 \%$ gel and $0.5 \%$ gel, respectively. For each image, the length of the second polarizing pulse, $t_{p 2}$, has been chosen so that the polarization of one of the images vanishes at the end of the inversion-recovery sequence. As $t_{p 2}$ is increased, each sample appears dark in succession, from shorter to longer $T_{1}$. Furthermore, in each image there is sufficient $T_{1}$-contrast to discriminate between the two non-vanishing columns. 


\section{SIMULTANEOUS MEG AND MRI}

Magnetoencephalography (MEG) is a noninvasive technique that measures tiny magnetic fields - typically $10^{-13} \mathrm{~T}$ to $10^{-11} \mathrm{~T}$ - generated by neural activity in the brain (29). These signals are detected by a large array of SQUID magnetometers or gradiometers, about 300 in state-of-the-art systems, arranged in a helmet containing liquid helium that is placed around the subject's head. Roughly 100 such systems are in use for both research and clinical applications, which include pre-surgical mapping of brain tumors and localization of the source of focal epilepsy. Although the temporal resolution of the SQUIDs - typically $1 \mathrm{~ms}$ - is much better than that of functional MRI (49), localization of the source requires solving the inverse problem, which generally has no unique solution. Maps of neural activity obtained from MEG are almost invariably combined with conventional MR images to correlate the magnetic sources with anatomical features or with functional MR images.

Combining MEG with direct imaging of the neural currents using low-field MRI would be a major step forward. Imaging neural currents requires the associated magnetic fields to cause some change in the NMR signal, for example, a change in frequency, phase or $T_{2}{ }^{*}$. Since these effects will undoubtedly be tiny, their observation presents a formidable challenge (50). A first step in this direction was the simultaneous measurement of an MEG signal and NMR free induction decay (FID) (51). The NMR measurement involved prepolarizing the subject's head in a field $B_{p} \approx 5 \mathrm{mT}$ for $1.5 \mathrm{sec}$ and observing the NMR signal at $6.3 \mu \mathrm{T}(268 \mathrm{~Hz})$ using a SQUID gradiometer. For the MEG measurement, a somatosensory response was evoked by applying an electrical current to the median nerve. During the prepolarization period, the SQUID was turned off. The SQUID feedback electronics was activated $10 \mathrm{~ms}$ after $B_{p}$ was removed, and data acquisition began $5 \mathrm{~ms}$ later. The stimulus was applied from 0 to $100 \mathrm{~ms}$ 
after $B_{p}$ was turned off. The FID and MEG signals were separated by means of $150-450 \mathrm{~Hz}$ and 3-100 Hz software filters, respectively.

Figure 14 shows the NMR signal and somatosensory response measured simultaneously. The stimulus was applied $100 \mathrm{~ms}$ after $B_{p}$ was turned off, introducing an artifact into the FID. The NMR signal arises from some relatively broad region of the cortex; no claim is made that this signal is correlated with the somatosensory response. Nonetheless, this experiment demonstrates the feasibility of combining MEG with microtesla NMR in a simultaneous measurement; extending this methodology to a 300-channel system should be possible.

\section{FUTURE OUTLOOK}

The system in this review has a system noise level of about $1 \mathrm{fT} \mathrm{Hz}^{-1 / 2}$. Implementing known improvements to the SQUID and gradiometer should reduce the noise to about $0.4 \mathrm{fT} \mathrm{Hz}^{-1 / 2}$ and perhaps to $0.2 \mathrm{fT} \mathrm{H}^{-1 / 2}$. The polarizing field is currently produced by a liquid nitrogencooled coil, which will shortly be replaced by a water-cooled coil that will enable longer averaging times. The combination of these two upgrades should lead to an improvement in the in-plane resolution of $2 \mathrm{~mm} \times 2 \mathrm{~mm}$ achieved on the forearm to perhaps $1 \mathrm{~mm} \times 1 \mathrm{~mm}$. It should be possible to achieve this resolution on limbs, so that microtesla MRI might be of interest in the imaging of joints of the arm, hand, leg and foot. A major advantage of this system is that it could undoubtedly be marketed for a substantially lower price than conventional MRI, thereby potentially lowering the cost of imaging significantly. Furthermore, the much lower weight and relatively small footprint imply that installation costs should be much lower than for high-field MRI. The open structure of the microtesla system - 
not to mention the low magnetic fields involved - should enable it to be used to monitor medical procedures.

Another potentially important imaging technique would be the use of a 300-SQUID helmet to image the brain just before or just after MEG. In principle, it would be relatively straightforward to add the necessary coils to an MEG system to perform microtesla MRI. Since magnetic source images are always superimposed on an MR image, the combined system could offer substantial cost savings. Furthermore, since the two kinds of images would be obtained with the same system in successive measurements, co-registering them would be particularly straightforward.

The unique strength of microtesla MRI, however, is the substantially higher $T_{1^{-}}$ weighted contrast achievable in microtesla fields. Although similar contrast has yet to be demonstrated in vivo, earlier ex vivo studies on healthy and cancerous breast tissue (47) suggest a substantial $T_{1}$-differentiation without the need for a contrast agent. Thus, a significant application might well be imaging of, for example, breast and prostate tumors. Breast tumors are well resolved in high-field MRI with the aid of a contrast agent, but the relatively high cost makes this technique too expensive for serial imaging to monitor the effects of therapy. Prostate tumors are not resolved in high-field $T_{1}$-weighted contrast images; $T_{2}$-weighted images (52) and spectroscopy (53) offer useful resolution, but are too expensive for routine use. Thus, if a relatively inexpensive microtesla MRI system can indeed image breast and prostate tumors, it could have significant impact on the monitoring of tumors for their response to therapy or during "watchful waiting" in the case of prostate cancer. The ability of this method to image in the presence of a metal tube suggests it could be used as a real-time monitor during biopsy. Another potential application is to monitor the insertion of 
radioactive "seeds" in prostate tumors (brachytherapy) (54). Cryosurgery involves the freezing of a tumor $(55,56)$. Microtesla MRI could monitor the insertion of the cryogenic tube and detect the onset of freezing: when the tissue solidifies, its $T_{l}$ drops dramatically, leading to the disappearance of the image. Since $T_{1}$ is temperature dependent, one might well be able to use microtesla MRI to monitor the temperature of tissue before it freezes; similarly, one could measure the temperature of a tumor and the surrounding tissue during radiotherapy (57).

Finally, there is the prospect of using microtesla MRI to image neural processes in real time. Such imaging presents enormous challenges, and indeed, it is not yet clear what modality - if any - could be used. Nonetheless, if such imaging could be achieved, it would offer new insights into neural activity.

\section{ACKNOWLEDGMENTS}

We gratefully acknowledge the contributions of Salvador Barriga, Sarah Busch, Erwin Hahn, Adam Hunt, Nathan Kelso, Darin Kinion, Peter Koo, SeungKyun Lee, Robert McDermott, Michael Mück, Alex Pines, Daniel Slichter, Bennie ten Haken and Andreas Trabesinger to the work described in this review. We thank Johannes Bernarding and Peter Volegov for supplying Figures 5(b) and 14, respectively. This work was supported by the Director, Office of Science, Office of Basic Energy Sciences, Materials Sciences and Engineering Division, of the U.S. Department of Energy under Contract No. DE-AC02-05CH11231. 


\section{LITERATURE CITED}

1. Edelman RR, Hesselink JR, Zlatkin MB, Crues JV. 2006. Clinical Magnetic Resonance Imaging, 3rd ed. Philadelphia: WB Saunders Company

2. Abragam A. 1961. The Principles of Nuclear Magnetism. London: Oxford University Press

3. Slichter CP. 1990. Principles of Nuclear Magnetic Resonance, $3^{\text {rd }}$ ed. New York: Springer-Verlag

4. Haacke EMR, Brown W, Thomson MR, Venkatesan R. 1999. Magnetic Resonance Imaging: Physical Principles and Sequence Design. New York: Wiley-Liss

5. Hu X, Norris DG. 2004. Advances in high-field magnetic resonance imaging. Annu. Rev. Biomed. Eng. 6:157-84

6. Macovski A, Conolly S. 1993. Novel approaches to low-cost MRI. Magn. Reson. Med. $30: 221-30$

7. Venook RD, Matter NI, Ramachandran M, Ungersma SE, Gold GE, et al. 2006. Prepolarized magnetic resonance imaging around metal orthopedic implants. Magn. Reson. Med. 56:177-86

8. Packard M, Varian R. 1954. Free nuclear induction in the Earth's magnetic field. Phys. Rev. 93:941-41

9. Stepisnik J, Erzen V, Kos M. 1990. NMR imaging in the earth's magnetic field. Magn. Reson. Med. 15:386-91

10. Happer W. 1972. Optical Pumping. Rev. Mod. Phys. 44:169-249

11. Tseng CH, Wong GP, Pomeroy VR, Mair RW, Hinton DP, et al. 1998. Low-field MRI of laser polarized noble gas. Phys. Rev. Lett. 81:3785-88 
12. Goodson BM. 2002. Nuclear magnetic resonance of laser-polarized noble gases in molecules, materials, and organisms. J. Magn. Reson. 155:157-216

13. Mair RW, Hrovat MI, Patz S, Rosen MS, Ruset IC, et al. ${ }^{3}$ He lung imaging in an open access, very-low-field human magnetic resonance imaging system. Magn. Reson. Med. $53: 745-49$

14. Venkatesh AK, Zhang AX, Mansour J, Kubatina L, Oh CH, et al. 2003. MRI of the lung gas-space at very low field using hyperpolarized noble gases. Magn. Reson. Imaging $21: 773-76$

15. Bidinosti CP, Choukeife J, Nacher PJ, Tastevin G. 2003. In vivo NMR of hyperpolarized 3He in the human lung at very low magnetic fields. J. Magn. Reson. 162:122-32

16. Clarke J, Braginski AI, ed. 2004 The SQUID Handbook Vol. 1. Weinheim: WileyVCH.

17. Greenberg Y. 1998. Application of superconducting quantum interference devices to nuclear magnetic resonance. Rev. Mod. Phys. 70:175-222

18. Seton HC, Hutchinson JMS, Bussel DM. 1997. A 4.2 K receiver coil and SQUID amplifier used to improve the SNR of low-field magnetic resonance images of the human arm. Meas. Sci. Tech. 8:198-207

19. Augustine MP, Wong-Foy A, Yarger JL, Tomaselli M, Pines A, et al. 1998. Low field magnetic resonance images of polarized noble gases obtained with a dc superconducting quantum interference device. Appl. Phys. Lett. 72:1908-10

20. Schlenga K, McDermott R, Clarke J, de Souza RE, Wong-Foy A, Pines A. 1999. Low-field magnetic resonance imaging with a high- $T_{c}$ dc superconducting quantum interference device. Appl. Phys. Lett. 75:3695-97 
21. McDermott R, Trabesinger AH, Mück M, Hahn EL, Pines A, et al. 2002. Liquid-state NMR and scalar couplings in microtesla magnetic fields. Science 295:2247-49

22. McDermott R, Lee S-K, ten Haken B, Trabesinger AH, Pines A, et al. 2004. Microtesla MRI with a superconducting quantum interference device. PNAS 7857-61

23. Matlachov AN, Volegov PL, Espy MA, George RS, Kraus RH. 2004. SQUID detected NMR in microtesla fields. J. Magn. Reson. 170:1-7

24. Burghoff M, Hartwig S, Trahms L, Bernarding J. 2005. Nuclear magnetic resonance in the nanoTesla range. Appl. Phys. Lett. 87:054103-1-3

25. McDermott R, Kelso N, Lee S-K, Mößle M, Mück M, et al. 2004. SQUID-detected magnetic resonance imaging in microtesla magnetic fields. J. Low Temp. Phys. 135:793821

26. Mößle M, Myers WR, Lee S-K, Kelso N, Hatridge M, et al. 2005. SQUID-detected in vivo MRI at microtesla magnetic fields. IEEE Trans. Appl. Supercond. 15:757-60

27. Yashchuk VV, Granwehr J, Kimball DF, Rochester SM, Trabesinger AH, et al. 2004. Hyperpolarized xenon nuclear spins detected by optical atomic magnetometry. Phys. Rev. Lett. 93:160801-1-4

28. Savukov M, Romalis MV. 2005. NMR detection with an atomic magnetometer. Phys. Rev. Lett. 94:123001-1-4

29. Vrba J, Nenone J, Trahms L. 2006. Biomagnetism. In The SQUID Handbook Vol. 2, ed. J Clarke, AI Braginski. Weinheim: Wiley-VCH

30. Tinkham M. 1996. Superconductivity. New York: McGraw Hill

31. Tesche CD, Clarke J. 1977. DC SQUID: noise and optimization. J. Low Temp. Phys. 29:301-31 
32. Hahn EL. 1950. Spin echoes. Phys. Rev. 80:580-94

33. Trabesinger AH, McDermott R, Lee, S-K, Mück M, Clarke J, Pines, A. 2004. SQUIDdetected liquid state NMR in microtesla fields. J. Phys. Chem. 108:957-63

34. Bernarding J, Buntkowsky G, Macholl S, Hartwig S, Burghoff M, et al. 2006. J-coupling nuclear magnetic resonance spectroscopy of liquids in nT fields. J. Am. Chem. Soc. $128: 714-15$

35. Bork J, Hahlbohm HD, Klein R, Schnabel A. 2001. The 8-layered magnetically shielded room of the PTB: Design and construction. In Biomag2000, Proc. 12th Int. Conf. on Biomagnetism, ed. J Nenonen, RJ Ilmoniemi, T Katila, pp. 970-73. Helsinki Univ. of Technology, Espoo, Finland

36. Myers WR. 2006. Potential applications of microtesla magnetic resonance imaging detected using a superconducting quantum interference device. $\mathrm{PhD}$ thesis. Univ. Calif., Berkeley. 192 pp.

37. Seton HC, Bussell DM, Hutchison JMS. 1998. U.K. patent numbers GB2331798 and GB2351549

38. Ludecke KM, Roschmann P, Tischler R. 1985. Susceptibility artefacts in NMR imaging. Magn. Reson. Imag. 3:329-343

39. Schenck JF. 1996. The role of magnetic susceptibility in magnetic resonance imaging: MRI magnetic compatibility of the first and second kinds. Medical Physics 23:815-50

40. Camacho CR, Plewes DB, Henkelmann RM. 1995. Nonsusceptibility artifacts due to metallic objects in MR imaging. J. Magn Reson. Imag. 5:75-88

41. Mößle M, Han S-I, Myers WR, Lee S-K, Kelso N, et al. 2006. SQUID-detected microtesla MRI in the presence of metal. J. Magn. Reson. 179:146-51 
42. Schnall, MD. 2003. Breast MR imaging. Radiol. Clin. N. Am. 41:43-50

43. Kaiser WA, Zeitler E. 1989. MR imaging of the breast: fast imaging sequences with and without Gd-DTPA. Preliminary observations. Radiology 170:681-86

44. Heywang, SH, Wolf A, Pruss AE, Hilbertz T, Eiermann W, Permanetter W. 1989. MR imaging of the breast with Gd-DTPA: use and limitations. Radiology 171:95-103

45. Mansfield P, Morris PG. 1982. NMR imaging in biomedicine. London: Academic Press

46. Lee S-K, Mößle M, Myers WR, Kelso N, Trabesinger AH, et al. 2005. SQUIDdetected MRI at $132 \mu \mathrm{T}$ with $T_{1}$-weighted contrast established at $10 \mu \mathrm{T}-300 \mathrm{mT}$. Magn Reson Med. 53:9-14

47. Koenig SH, Brown RD III. 1987. Relaxation of tissue. In NMR Spectroscopy of Cells and Organisms, Vol. II, ed. RK Gupta, pp.75-114. Boca Raton: CRC Press

48. Planinsic G, Stepisnik J, Kos M. 1994. Relaxation-time measurement and imaging in the Earth's magnetic field. J. Magn. Reson. 110:170-74

49. Huettel SA, Song AW, McCarthy G. 2004. Functional magnetic resonance imaging. Sunderland: Sinauer Associates

50. Bodurka J, Bandettini PA. 2002. Toward direct mapping of neuronal activity: MRI detection of ultraweak, transient magnetic field changes. Magn. Reson. Med. 47:1052-58.

51. Volegov P, Matlachov AN, Espy MA, George JS, Kraus Jr. RH. 2004. Simultaneous magnetoencephalography and SQUID detected nuclear MR in microtesla magnetic fields. Magn Reson Med. 52:467-70

52. Claus FG, Hricak H, Hattery RR. 2004. Pretreatment evaluation of prostate cancer: role of MR Imaging and 1HMR spectroscopy. Radiographics 24:S167-80 
53. Kurhanewicz J, Swanson MG, Nelson SJ, Vigneron DB. 2002. Combined magnetic resonance imaging and spectroscopic imaging approach to molecular imaging of prostate cancer. J. Magn Reson. Imag. 16:451-63

54. Susil RC, Camphausen K, Choyke P, McVeigh ER, Gustafson GS, et al. 2004. System for prostate brachytherapy and biopsy in a standard 1.5 T MRI scanner. Magn. Reson. Med. $52: 683-87$

55. Pease GR, Wong ST, Roos MS, Rubinsky B. 1995. MR image-guided control of cryosurgery. J. Magn. Reson. Imag 5:753-60

56. Onik G. 2001. Image-guided prostate cryosurgery: state of the art. Cancer Control 8:52231

57. Hynynen K, McDannold N. 2004. MRI guided and monitored focused ultrasound thermal ablation methods: a review of progress. Int. J. Hyperthermia. 20:725-37 


\title{
Key Words
}

Superconducting QUantum Interference Device, nuclear magnetic resonance, magnetic resonance imaging, longitudinal-relaxation-time-weighted contrast imaging

\begin{abstract}
The use of very low noise magnetometers based on Superconducting QUantum Inteference Devices (SQUIDs) enables nuclear magnetic resonance (NMR) and magnetic resonance imaging (MRI) in microtesla magnetic fields. An untuned superconducting flux transformer coupled to a SQUID achieves a magnetic field noise of $10^{-15} \mathrm{THz}^{-1 / 2}$. The frequencyindependent response of this magnetometer combined with prepolarization of the nuclear spins yields an NMR signal that is independent of the Larmor frequency $\omega_{0}$. An MRI system operating in a field of $132 \mu \mathrm{T}$, corresponding to a proton frequency of $5.6 \mathrm{kHz}$, achieves an inplane resolution of $0.7 \times 0.7 \mathrm{~mm}^{2}$ in phantoms. Measurements of the longitudinal relaxation time $T_{1}$ in different concentrations of agarose gel over five decades of frequency reveal much greater $T_{1}$-differentiation at fields below a few millitesla. Microtesla MRI has the potential to image tumors with substantially greater $T_{1}$-weighted contrast than is achievable in high fields in the absence of a contrast agent.
\end{abstract}




\section{List of definitions and acronyms}

MRI: magnetic resonance imaging

NMR: nuclear magnetic resonance

$B_{0}$ : precession field, the magnetic field in which nuclear spins precess at their Larmor frequency during data acquisition.

$\gamma:$ gyromagnetic ratio

SNR: signal-to-noise ratio

$B_{p}$ : prepolarizing magnetic field

SQUID: Superconducting QUantum Interference Device - an ultrasensitive detector of magnetic flux

$T_{1}$ : longitudinal relaxation time - the characteristic time with which nuclear spins in a magnetic field relax to thermal equilibrium after being perturbed

$T_{2}$ : transverse relaxation time - the characteristic time with which precessing nuclear spins dephase due to spin-spin interactions

$T_{2}^{\prime}$ : the characteristic time with which precessing nuclear spins dephase due to inhomogeneities in the precession field

$T_{2}^{*}=\left(1 / T_{2}+1 / T_{2}^{\prime}\right)^{-1}$ 
$T_{1}$-weighted contrast image: MR image in which each voxel is weighted according to its value of $T_{1}$. This technique is widely used in MRI to differentiate different tissue types.

MEG: magnetoencephalography - the detection of spontaneous or stimulated magnetic fields from the human brain by means of arrays of SQUIDs (as many as 300) arranged around the head in a "helmet" containing liquid helium.

$\Phi_{0}:$ flux quantum $\mathrm{h} / 2 \mathrm{e} \approx 2.07 \times 10^{-15} \mathrm{Tm}^{2}$. 


\section{SUMMARY POINTS}

1. The SQUID is an ultrasensitive detector of magnetic flux. Coupled to a superconducting flux transformer, it has a typical sensitivity of $1 \mathrm{fTHz}^{-1 / 2}$. The response of a SQUID coupled to an untuned flux transformer is independent of frequency.

2. In conventional NMR and MRI with a Faraday detector, the amplitude of the signal scales as $B_{0}{ }^{2}$, where $B_{0}$ is the Larmor field. In contrast, for a SQUID, the amplitude scales as $B_{0}$; if the spins are prepolarized at a field higher than $\mathrm{B}_{0}$, the amplitude is independent of $\mathrm{B}_{0}$.

3. For a given relative magnetic field inhomogeneity $\Delta B_{0} / B_{0}$, the NMR inhomogeneous linewidth decreases as $B_{0}$ is reduced until it becomes limited by the homogeneous linewidth.

4. The response of a flux transformer configured as a second-derivative gradiometer scales as $1 / r^{5}$, where $r$ is the distance to a fluctuating magnetic dipole. The use of such gradiometers greatly attenuates spurious magnetic field noise.

5. At an imaging field of $132 \mu \mathrm{T}, 2-\mathrm{D}$ images of a water phantom and 3-D images of a pepper and a forearm demonstrate in-plane resolution of $0.7 \times 0.7 \mathrm{~mm}^{2}, 2 \times 2 \mathrm{~mm}^{2}$ and $2 \times 2 \mathrm{~mm}^{2}$, respectively.

6. Compared with high fields, imaging in low magnetic fields greatly reduces the image distortion introduced by the presence of nonmagnetic metals.

7. At magnetic fields below a few millitesla, the $T_{1}$-contrast of phantoms containing different concentrations of agarose gel is substantially enhanced over that at higher fields. Images of agarose gel phantoms acquired with $T_{1}$-weighted contrast at low fields correspondingly showed much higher differentiation of these different concentrations compared with those in high fields. 
8. A promising clinical application of microtesla MRI is to image tumors, for example, breast and prostate tumors, with greater $T_{1}$-contrast than is possible with high-field MRI in the absence of a contrast agent. 


\section{ANNOTATED REFERENCES}

(1) Comprehensive review of the current state-of-the-art of clinical MRI

$(2,3)$ Two classic references on all aspects of nuclear magnetic resonance

(4) Comprehensive review of the physical principles underlying MRI, with extensive discussion of pulse sequences used in a wide variety of imaging modalities.

(16) Comprehensive overview of the principles of SQUIDs, with 7 chapters by different authors describing the principles, theory, design, fabrication and operation of SQUIDs.

(17) Comprehensive review of SQUID-based NMR and MRI through 1997, discussing approximately 100 such experiments.

(18) Paper describing the use of SQUIDs with a superconducting tuned input circuit to perform MRI at $425 \mathrm{kHz}$.

(21) First paper on microtesla NMR performed with a SQUID with a superconducting untuned input circuit. This paper demonstrates the narrowing of the NMR linewidth achieved by reducing the magnitude of an inhomogeneous magnetic field, and shows spectra of Jcoupled nuclei.

(22) First paper on MRI in microtesla fields, showing 2-D images of peppers.

(46) Paper showing variation in $T_{1}$-contrast in two concentrations of agarose gel over five decades of frequency. Presents $T_{1}$-contrast images of gel samples and water, including those obtained with inversion recovery. 


\section{FIGURE CAPTIONS}

Figure 1 Principle of the SQUID. (a) Schematic showing two Josephson junctions (marked "x") connected in parallel on a superconducting loop. Above a threshold value, the current $I$ induces a voltage $V$ across the SQUID. (b) Current-voltage characteristic for integer (n) and half-integer $(n+1 / 2)$ values of applied flux $\Phi$ threading the SQUID loop. (c) Voltage $V$ across the SQUID versus applied flux (in units of the flux quantum) for fixed bias current through the SQUID. A small change in applied flux $\delta \Phi$ produces a voltage change $\delta V$.

Figure 2 Practical devices. (a) Photograph of a thin-film SQUID fabricated at Berkeley (right) and a close up of the Josephson junction area (left) (b) Configuration of a flux transformer coupled to a SQUID to form a magnetometer.

Figure 3 Representative magnitudes of magnetic fields on a logarithmic scale.

Figure 4 NMR spectra of mineral oil. (a) Spectrum averaged over 10,000 acquisitions with $B_{0}=1.8 \mathrm{mT}$. (b) Spectrum averaged over 100 acquisitions with $B_{\mathrm{p}}=$ $1.8 \mathrm{mT}, B_{0}=1.8 \mu \mathrm{T}$.

Figure 5 NMR spectra of J-coupled nuclei in microtesla fields. (a) Spectrum of trimethyl phosphate acquired with $B_{0}=4.8 \mu \mathrm{T}$. (b) Spectrum of 2, 2, 2-trifluorethanol acquired with $B_{0}=993 \mathrm{nT}$. [Reprinted with permission from (34). Copyright 2006 American Chemical Society]

Figure 6 Configuration of SQUID MRI system. (a) Schematic of magnetic field coils, gradient coils ( $G_{y}$ omitted for clarity) sample, and dewar containing detector assembly. (b) Photograph of magnetic field and field gradient coil assembly. 
Figure 7 Schematic of SQUID and second-derivative gradiometer showing series array of Josephson junctions.

Figure 8 Pulse sequence for three-dimensional imaging.

Figure 9 Two- and three-dimensional MR images. (a) Two-dimensional image of water in a multi-well plate, acquired with $B_{0}=132 \mu \mathrm{T}$, field gradient $=240 \mu \mathrm{T} / \mathrm{m}$ and $B_{p}$ $=85 \mathrm{mT}$. The in-plane resolution is $0.7 \mathrm{~mm} \times 0.7 \mathrm{~mm}$. (b) Three-dimensional image of a whole bell pepper, acquired with $B_{0}=132 \mu \mathrm{T}$, field gradient $=120 \mu \mathrm{T} / \mathrm{m}$ and $B_{p}=60$ $\mathrm{mT}$. The in-plane resolution is $1.9 \mathrm{~mm} \times 1.6 \mathrm{~mm}$, slice thickness is $10 \mathrm{~mm}$.

Figure 10 Three-dimensional in vivo image of a human forearm. Image was acquired with $B_{0}=132 \mu \mathrm{T}$, field gradient $=150 \mu \mathrm{T} / \mathrm{m}$ and $B_{p}=40 \mathrm{mT}$. The in-plane resolution is $2 \mathrm{~mm} \times 2 \mathrm{~mm}$, slice thickness is $20 \mathrm{~mm}$. Slices (a)-(d) progress from the wrist to the elbow.

Figure 11 Images of water phantoms containing a $1.5 \mathrm{~mm} \times 10 \mathrm{~mm} \times 15 \mathrm{~mm}$ bar of titanium. (a) Photograph of high field phantom. (b) and (c) Images of high field phantom with the long axis of the titanium bar parallel and perpendicular to the frequency encoding gradient. The images were acquired with $B_{0}=7 \mathrm{~T}$ and $G_{\text {freq }}=40$ $\mathrm{mT} / \mathrm{m}$. (d) Photograph of low field phantom. (e) Image of low field phantom, acquired with $B_{0}=66 \mu \mathrm{T}, G_{\text {freq }}=110 \mu \mathrm{T} / \mathrm{m}$ and $G_{p}=85 \mathrm{mT}$.

Figure 12 Low-field $T_{1}$ contrast of agarose gel and water. (a) Pulse sequence used for $T_{1}$-weighted imaging and acquisition of $T_{1}$-dispersion curves. (b) $T_{1}$-dispersion curves for water and two different concentrations of agarose gel. (c) Images of a phantom consisting of $0.5 \%$ agarose gel and 1 to $6 \mathrm{~mm}$-diameter columns of water with $T_{1}$-contrast weighted at $100 \mathrm{mT}(\mathrm{A})$ and $132 \mu \mathrm{T}(\mathrm{B})$. 
Figure $13 T_{1}$-weighted contrast imaging with inversion recovery. (a) Pulse sequence showing inversion recovery technique. (b) Schematic of a phantom containing two concentrations of agarose gel and pure water. (c) Images of the phantom with constant $t_{p 1}=2 \mathrm{~s}$ and $t_{\mathrm{int}}=200 \mathrm{~ms}$, and variable $t_{p 2}$ of $200 \mathrm{~ms}, 700 \mathrm{~ms}$, and $900 \mathrm{~ms}$, respectively, demonstrating selective nulling of each of the agarose gel concentrations and water.

Figure 14 Simultaneous ${ }^{1} \mathrm{H}$ NMR FID (blue trace) and MEG somatosensory response (red trace) acquired from a region of the human head including the somatosensory cortex. [Reprinted with permission from (51)] 